\title{
Multifarious results for q-Hermite based Frobenius type Eulerian polynomials
}

\author{
Waseem Ahmad Khan ${ }^{1}$, Idrees Ahmad Khan ${ }^{2}$, Mehmet Acikgoz ${ }^{3}$ and Ugur Duran ${ }^{4, *}$ \\ ${ }^{1}$ Department of Mathematics, Faculty of Science, \\ Integral University, Lucknow-226026, India \\ E-Mail: waseem08 khan@rediffmail.com \\ ${ }^{2}$ Department of Mathematics, Faculty of Science, \\ Integral University, Lucknow-226026, India \\ E-Mail: khanidrees077@gmail.com \\ ${ }^{3}$ University of Gaziantep, Faculty of Science and Arts, \\ Department of Mathematics, TR-27310 Gaziantep, Turkey \\ E-Mail: acikgoz@gantep.edu.tr \\ ${ }^{4}$ Department of the Basic Concepts of Engineering, \\ Faculty of Engineering and Natural Sciences, \\ Iskenderun Technical University, TR-31200 Hatay, Turkey \\ E-Mail: mtdrnugur@gmail.com \& ugur.duran@iste.edu.tr \\ ${ }^{*}$ Corresponding Author
}

\begin{abstract}
In this paper, a new class of $q$-Hermite based Frobenius type Eulerian polynomials is introduced by means of generating function and series representation. Several fundamental formulas and recurrence relations for these polynomials are derived via different generating methods. Furthermore, diverse correlations including the $q$-Apostol-Bernoulli polynomials, the $q$-Apostol-Euler poynoomials, the $q$-Apostol-Genocchi polynomials and the $q$-Stirling numbers of the second kind are also established by means of the their generating functions.
\end{abstract}

2010 Mathematics Subject Classification. Primary 11B73, Secondary 11B83, 11B68.

Key Words and Phrases. Hermite polynomials, Frobenius type Eulerian polynomials, Hermite based Frobenius type Eulerian polynomials, $q$-numbers, $q$-polynomials.

\section{INTRODUCTION}

The subject of $q$-calculus started appearing in the nineteenth century due to its applications in various fields of mathematics, physics and engineering. One of the most popular studies in $q$-calculus is the $q$ extension of the some special polynomials such as Bernoulli, Eulerian, Genocchi, Euler polynomials, $c f$. [1-19] and see also the references cited therein. The classical Eulerian polynomials are firstly considered by Leonhard Euler in his Remarques sur un beau rapport entre les séries des puissances tant directes que réciproques in 1749 (first printed in 1765) inwhich he defines a method of computing values of the zeta function at negative integers. Then, the foregoing polynomials have been studied and investigated extensively until now, cf. $[3,4,6,7]$. Moreover, the Frobenius-type Eulerian polynomials are introduced and studied in [19]. In this paper, we perform to give a novel class of $q$-generalization of the Hermite based Frobenius type Eulerian polynomials and to derive multifarious correlation, implicit summation formula, identities, explicit formulas and recurrence relations for the mentioned polynomials by means of the series manipulation 
methods. Furthermore, we investigate some correlations covering the $q$-Apostol-Bernoulli polynomials, $q$ Apostol-Euler polynomials, $q$-Apostol-Genocchi polynomials and $q$-Stirling numbers of the second kind for the $q$-Hermite based Frobenius type Eulerian polynomials.

The definitions and notations of $q$-calculus reviewed here are taken from the references $[5,8-13,16]$ :

The $q$-analogue of the shifted factorial $(a)_{n}$ is given by

$$
(a ; q)_{0}=1,(a ; q)_{n}=\prod_{m=0}^{n-1}\left(1-q^{m} a\right), \quad n \in \mathbb{N} .
$$

The $q$-analogues of a complex number $a$ and of the factorial function are given by

$$
[a]_{q}=\frac{1-q^{a}}{1-q}, q \in \mathbb{C} ; 0<|q|<1 ; a \in \mathbb{C},
$$

and

$$
[n]_{q} !=\prod_{m=1}^{n}[m]_{q}=[1]_{q}[2]_{q} \cdots[n]_{q}=\frac{(q ; q)_{n}}{(1-q)^{n}} ; \quad n \in \mathbb{N}, q \in \mathbb{C} ; 0<|q|<1
$$

with $[0]_{q} !=1$. The Gauss $q$-binomial coefficient $\left(\begin{array}{l}n \\ k\end{array}\right)_{q}$ is given by

$$
\left(\begin{array}{l}
n \\
k
\end{array}\right)_{q}=\frac{[n]_{q} !}{[k]_{q} ![n-k]_{q} !}=\frac{(q ; q)_{n}}{(q ; q)_{k}(q ; q)_{n-k}}, k=0,1, \cdots, n .
$$

The $q$-analogue of the function $(x+y)_{q}^{n}$ is given by

$$
(x+y)_{q}^{n}=\sum_{k=0}^{n}\left(\begin{array}{l}
n \\
k
\end{array}\right)_{q} q^{k(k-1) / 2} x^{n-k} y^{k}, n \in \mathbb{N}_{0} .
$$

The $q$-analogues of exponential function are given by

$$
e_{q}(x)=\sum_{n=0}^{\infty} \frac{x^{n}}{[n]_{q} !}=\frac{1}{((1-q) x ; q)_{\infty}}, 0<|q|<1 ;|x|<|1-q|^{-1}
$$

and

$$
E_{q}(x)=\sum_{n=0}^{\infty} q^{n(n-1) / 2} \frac{x^{n}}{[n]_{q} !}=(-(1-q) x ; q)_{\infty}, 0<|q|<1 ; x \in \mathbb{C} .
$$

Moreover, the functions $e_{q}(x)$ and $E_{q}(x)$ satisfy the following properties:

$$
D_{q} e_{q}(x)=e_{q}(x), D_{q} E_{q}(x)=E_{q}(q x),
$$

where the $q$-derivative $D_{q} f$ of a function $f$ at a point $0 \neq z \in \mathbb{C}$ is defined as follows:

$$
D_{q} f(z)=\frac{f(q z)-f(z)}{q z-z}, 0<|q|<1
$$

For any two arbitrary functions $f(z)$ and $g(z)$, the $q$-derivative operator $D_{q}$ satisfies the following product and quotient relations:

$$
D_{q, z}(f(z) g(z))=f(z) D_{q, z} g(z)+g(q z) D_{q, z} f(z)
$$

and

$$
D_{q, z}\left(\frac{f(z)}{g(z)}\right)=\frac{g(q z) D_{q, z} f(z)-f(q z) D_{q, z} g(z)}{g(z) g(q z)} .
$$


The Apostol type $q$-Bernoulli polynomials $B_{n, q}^{(\alpha)}(x, y ; \lambda)$ of order $\alpha$, the Apostol type $q$-Euler polynomials $E_{n, q}^{(\alpha)}(x, y ; \lambda)$ of order $\alpha$ and the Apostol type $q$-Genocchi polynomials $G_{n, q}^{(\alpha)}(x, y ; \lambda)$ of order $\alpha$ are defined by means of the following generating function (see [8-13]):

$$
\begin{gathered}
\left(\frac{t}{\lambda e_{q}(t)-1}\right)^{\alpha} e_{q}(x t) E_{q}(y t)=\sum_{n=0}^{\infty} B_{n, q}^{(\alpha)}(x, y ; \lambda) \frac{t^{n}}{n !},(|t+\log \lambda|)<2 \pi, 1^{\alpha}=1 \\
\left(\frac{2}{\lambda e_{q}(t)+1}\right)^{\alpha} e_{q}(x t) E_{q}(y t)=\sum_{n=0}^{\infty} E_{n, q}^{(\alpha)}(x, y ; \lambda) \frac{t^{n}}{n !},(|t+\log \lambda|)<\pi, 1^{\alpha}=1
\end{gathered}
$$

and

$$
\left.\left(\frac{2 t}{\lambda e_{q}(t)+1}\right)^{\alpha} e_{q}(x t) E_{q}(y t)=\sum_{n=0}^{\infty} G_{n, q}^{(\alpha)}(x, y ; \lambda) \frac{t^{n}}{n !},|t+\log \lambda|\right)<\pi, 1^{\alpha}=1 .
$$

Clearly, we have

$$
B_{n, q}^{(\alpha)}(\lambda)=B_{n, q}^{(\alpha)}(0,0 ; \lambda), E_{n, q}^{(\alpha)}(\lambda)=E_{n, q}^{(\alpha)}(0,0 ; \lambda), G_{n, q}^{(\alpha)}(\lambda)=G_{n, q}^{(\alpha)}(0,0 ; \lambda) .
$$

The Frobenius-type Eulerian polynomials $A_{n}^{(\alpha)}(x ; \lambda)$ of order $\alpha \in \mathbb{C}$ are defined by means of the following generating function as follows (see $[3,4,6,7]$ ):

$$
\left(\frac{1-\lambda}{e^{t(\lambda-1)}-\lambda}\right)^{\alpha} e^{x t}=\sum_{n=0}^{\infty} A_{n}^{(\alpha)}(x ; \lambda) \frac{t^{n}}{n !},
$$

where $\lambda$ is a complex number with $\lambda \neq 1$. The number $A_{n}$ is given by

$$
A_{n}^{(\alpha)}(\lambda)=A_{n}^{(\alpha)}(0 ; \lambda)
$$

are called the Frobenius type Eulerian numbers (see [17, 19]). Clearly, we have

$$
A_{n}^{(\alpha)}(x ; \lambda)=\sum_{m=0}^{n}\left(\begin{array}{c}
n \\
m
\end{array}\right) A_{m}^{(\alpha)}(\lambda) x^{n-m} .
$$

In recent days, a new type of $q$-Hermite polynomials are considered in $[12,16]$, which is a particular member of the $q$-Appell family [1]. The $q$-Appell polynomials are defined by means of the following generating function:

$$
\frac{1}{g_{q}(t)} e_{q}(x t)=\sum_{n=0}^{\infty} A_{n, q}(x) \frac{t^{n}}{[n]_{q} !}, A_{n, q}=A_{n, q}(0) .
$$

Definition 1.1. The continuous $q$-Hermite polynomials $H_{n, q}^{(s)}(x)$ are defined by

$$
e_{q}\left(x t-\frac{s t^{2}}{1+q}\right)=\sum_{n=0}^{\infty} H_{n, q}^{(s)}(x) \frac{t^{n}}{[n]_{q} !},(0<q<1,0 \neq s \in \mathbb{R})
$$

where $H_{n, q}^{(s)}=H_{n, q}^{(s)}(0)$ are the continuous $q$-Hermite numbers defined by

$$
e_{q}\left(\frac{s t^{2}}{1+q}\right)=\sum_{n=0}^{\infty} H_{n, q}^{(s)}(0) \frac{t^{n}}{[n]_{q} !} \text {. }
$$

\section{2. $q$-Hermite-based Frobenius type Eulerian polynomials ${ }_{H} A_{n, q}^{(\alpha, s)}(x, y ; \lambda)$}

In this section, we define $q$-Hermite-based Frobenius type Eulerian polynomials $(q H b F t E p)_{H} A_{n, q}^{(\alpha, s)}(x, y ; \lambda)$ by means of the generating function and series representation. Certain relations for these polynomials are also derived by using various identities. We now ready to start in conjunction with the following definition. 
Definition 2.1. Let $q, \lambda \in \mathbb{C}, \alpha \in \mathbb{N}, 0<|q|<1$. The generalized $q$-Hermite-based Frobenius type Eulerian polynomials ${ }_{H} A_{n, q}^{(\alpha, s)}(x, y ; \lambda)$ of order $\alpha$ are defined by means of the following generating function:

$$
\left(\frac{1-\lambda}{e_{q}^{t(\lambda-1)}-\lambda}\right)^{\alpha} e_{q}\left(x t-\frac{s t^{2}}{1+q}\right) E_{q}(y t)=\sum_{n=0}^{\infty}{ }_{H} A_{n, q}^{(\alpha, s)}(x, y ; \lambda) \frac{t^{n}}{[n]_{q} !}
$$

When $x=y=s=0$ in $(2.1),{ }_{H} A_{n}^{(\alpha, 0)}(0,0 ; \lambda):=A_{n}^{(\alpha)}(\lambda)$ are called the $n^{t h}$ Frobenius type Eulerian numbers of order $\alpha$.

Remark 2.1. For $x=y=0$ in $(2.1),{ }_{H} A_{n, q}^{(\alpha, s)}(\lambda)={ }_{H} A_{n, q}^{(\alpha, s)}(0,0 ; \lambda)$ are the the $q$-Hermite based Frobenius type Eulerian numbers defined by

$$
\left(\frac{1-\lambda}{e_{q}^{t(\lambda-1)}-\lambda}\right)^{\alpha} e_{q}\left(\frac{s t^{2}}{1+q}\right)=\sum_{n=0}^{\infty} H A_{n, q}^{(\alpha, s)}(\lambda) \frac{t^{n}}{[n]_{q} !} .
$$

Theorem 2.1. The following identity for ${ }_{H} A_{n, q}^{(\alpha, s)}(x, y ; \lambda)$

$$
(\lambda-1)^{-n}{ }_{H} A_{n, q}^{\left.\left(\alpha,(\lambda-1)^{2} s\right)\right)}\left((\lambda-1) x,(\lambda-1)^{2} y ; \lambda\right)={ }_{H} H_{n, q}^{(\alpha, s)}(x, y ; \lambda)
$$

holds true.

Proof. Consider the generating function (2.1), we have

$$
\begin{gathered}
\sum_{n=0}^{\infty} \frac{H_{n, q}^{\left(\alpha,(\lambda-1)^{2} s\right)}((\lambda-1) x,(\lambda-1) y ; \lambda)}{n !} \frac{t^{n}}{(\lambda-1)^{n}} \\
=\left(\frac{1-\lambda}{e_{q}(t)-\lambda}\right)^{\alpha} e_{q}\left(x t-\frac{s t^{2}}{1+q}\right) E_{q}(y t)=\sum_{n=0}^{\infty} H_{n, q}^{(\alpha, s)}(x, y ; \lambda) \frac{t^{n}}{[n]_{q} !},
\end{gathered}
$$

where ${ }_{H} H_{n, q}^{(\alpha, s)}(x, y ; \lambda)$ are called the $q$-Hermite based Frobenius polynomials, which is defined by Riyasat and Khan [16] and comparing the coefficients of $t^{n}$, we arrive at the required result (2.3).

Theorem 2.2. The following summation representation for the $q$-Hermite-based Frobenius type Eulerian polynomials ${ }_{H} A_{n, q}^{(\alpha, s)}(x, y ; \lambda)$ of order $\alpha$

$$
{ }_{H} A_{n, q}^{(\alpha, s)}(x, y ; \lambda)=\sum_{m=0}^{n}\left(\begin{array}{c}
n \\
m
\end{array}\right) A_{q}^{(\alpha)}(y, \lambda)(\lambda) H_{m, q}^{(s)}(x)
$$

holds true.

Proof. Using equation (1.10) and (1.12) in the left hand side of equation (2.1) and then applying the Cauchy product rule and equating the coefficients of same powers of $t$ in both sides of resultant equation, we get representation $(2.4)$.

Theorem 2.3. The following summation formulae

$$
\begin{gathered}
{ }_{H} A_{n, q}^{(\alpha, s)}(x, y ; \lambda)=\sum_{m=0}^{n}\left(\begin{array}{c}
n \\
m
\end{array}\right)_{q}{ }_{H} A_{m, q}^{(\alpha, s)}(0,0 ; \lambda)(x+y)_{q}^{n-m}, \\
{ }_{H} A_{n, q}^{(\alpha, s)}(x, y ; \lambda)=\sum_{m=0\left(\begin{array}{l}
n \\
m
\end{array}\right)_{q} H}^{n} A_{m, q}^{(\alpha, s)}(0, y ; \lambda) x^{n-m}
\end{gathered}
$$

and

$$
{ }_{H} A_{n, q}^{(\alpha, s)}(x, y ; \lambda)=\sum_{m=0}^{n}\left(\begin{array}{l}
n \\
m
\end{array}\right) q_{q}(n-k)(n-k-1) / 2{ }_{H} A_{m, q}^{(\alpha, s)}(x, 0 ; \lambda) y^{n-m}
$$

hold true for the $q$-Hermite-based Frobenius type Eulerian polynomials of order $\alpha$. 
Proof. Suitably using equations (1.1)-(1.3) in generating function (2.1) to get three different form. Further making use of the Cauchy product rule in the resultant expressions and then comparing the like powers of $t$ in the both sides of resultant equation, we find formulas (2.5)-(2.7).

Theorem 2.4. The following recursive formulas for the $q$-Hermite-based Frobenius type Eulerian polynomials ${ }_{H} A_{n, q}^{(\alpha, s)}(x, y ; \lambda)$ of order $\alpha$ holds true:

$$
D_{q, x H} A_{n, q}^{(\alpha, s)}(x, y ; \lambda)=[n]_{q H} A_{n-1, q}^{(\alpha, s)}(x, y ; \lambda)
$$

and

$$
D_{q, y H} A_{n, q}^{(\alpha, s)}(x, y ; \lambda)=[n]_{q H} A_{n-1, q}^{(\alpha, s)}(x, q y ; \lambda) .
$$

Proof. Differentiating generating function (2.1) with respect to $x$ and $y$ with the help of equation (1.4) and then simplifying with the help of the Cauchy product rule formulas (2.8) and (2.9) are obtained.

Theorem 2.5. The following relation for the $q$-Hermite-based Frobenius type Eulerian polynomials ${ }_{H} A_{n, q}^{(\alpha, s)}(x, y ; ; \lambda)$ of order $\alpha$ holds true:

$$
(2 \lambda-1) \sum_{k=0}^{n}\left(\begin{array}{l}
n \\
k
\end{array}\right)_{q} A_{k, q}(0, y ; \lambda)_{H} A_{n-k, q}^{(s)}(x, 0 ; 1-\lambda)=\lambda_{H} A_{n, q}^{(s)}(x, y ; \lambda)-(1-\lambda)_{H} A_{n, q}^{(s)}(x, y ; 1-\lambda) .
$$

Proof. We set

From the above equation, we see that

$$
\frac{(2 \lambda-1)}{\left(e_{q}^{t(\lambda-1)}-\lambda\right)\left(e_{q}^{t(\lambda-1)}-(1-\lambda)\right)}=\frac{1}{e_{q}^{t(\lambda-1)}-\lambda}-\frac{1}{e_{q}^{t(\lambda-1)}-(1-\lambda)}
$$

$$
\begin{gathered}
(2 \lambda-1) \frac{(1-\lambda) e_{q}\left(x t-\frac{s t^{2}}{1+q}\right)(1-(1-\lambda)) E_{q}(y t)}{\left(e_{q}^{t(\lambda-1)}-\lambda\right)\left(e_{q}^{t(\lambda-1)}-(1-\lambda)\right)} \\
=\frac{(1-\lambda) e_{q}\left(x t-\frac{s t^{2}}{1+q}\right) \lambda E_{q}(y t)}{e_{q}^{t(\lambda-1)}-\lambda}-\frac{(1-\lambda) e_{q}\left(x t-\frac{s t^{2}}{1+q}\right) E_{q}(y t)(1-(1-\lambda))}{e_{q}^{t(\lambda-1)}-(1-\lambda)},
\end{gathered}
$$

which on using equations (1.10) and (2.1) in both sides, we have

$$
\begin{aligned}
& (2 \lambda-1)\left(\sum_{n=0}^{\infty}{ }_{H} A_{k, q}(0, y ; \lambda) \frac{t^{k}}{[k]_{q} !}\right)\left(\sum_{n=0}^{\infty}{ }_{H} A_{n, q}^{(s)}(x, 0 ; 1-\lambda) \frac{t^{n}}{[n]_{q} !}\right) \\
& =\lambda \sum_{n=0}^{\infty}{ }_{H} A_{n, q}^{(s)}(x, y ; \lambda) \frac{t^{n}}{[n]_{q} !}-(1-\lambda) \sum_{n=0}^{\infty} H_{H} A_{n, q}^{(s)}(x, y ; 1-\lambda) \frac{t^{n}}{[n]_{q} !} .
\end{aligned}
$$

Applying the Cauchy product rule in the above equation and then equating the coefficients of like powers of $t$ in both sides of the resultant equation, assertion (2.10) follows.

Theorem 2.6. The following relation for the $q$-Hermite-based Frobenius-type Eulerian polynomials ${ }_{H} A_{n, q}^{(\alpha, s)}(x, y ; \lambda)$ of order $\alpha$ holds true:

$$
\lambda_{H} A_{n, q}^{(s)}(x, y ; \lambda)=\sum_{k=0}^{n}\left(\begin{array}{l}
n \\
k
\end{array}\right)_{q}{ }_{q} A_{n-k, q}^{(s)}(x, y ; \lambda)(1-\lambda)^{k}-(1-\lambda) H_{n, q}^{(s)}(x, y) .
$$

Proof. Consider the following identity

$$
\frac{\lambda}{\left(e_{q}^{t(\lambda-1)}-\lambda\right) e_{q}^{t(\lambda-1)}}=\frac{1}{\left(e_{q}^{t(\lambda-1)}-\lambda\right)}-\frac{1}{e_{q}^{t(\lambda-1)}} .
$$

Evaluating the following fraction using above identity, we find

$$
\frac{\lambda(1-\lambda) e_{q}\left(x t-\frac{s t^{2}}{1+q}\right) E_{q}(y t)}{\left(e_{q}^{t(\lambda-1)}-\lambda\right) e_{q}^{t(\lambda-1)}}=\frac{(1-\lambda) e_{q}\left(x t-\frac{s t^{2}}{1+q}\right) E_{q}(y t)}{\left(e_{q}^{t(\lambda-1)}-\lambda\right)}-\frac{(1-\lambda) e_{q}\left(x t-\frac{s t^{2}}{1+q}\right) E_{q}(y t)}{e_{q}^{t(\lambda-1)}}
$$




$$
\lambda \sum_{n=0}^{\infty} H_{n, q}^{(s)}(x, y ; \lambda) \frac{t^{n}}{[n]_{q} !}=\sum_{n=0}^{\infty} H_{H} A_{n, q}^{(s)}(x, y ; \lambda) \frac{t^{n}}{n !} \sum_{k=0}^{\infty}(1-\lambda)^{k} \frac{t^{k}}{[k]_{q} !}-(1-\lambda) \sum_{n=0}^{\infty} H_{n, q}^{(s)}(x, y) \frac{t^{n}}{[n]_{q} !} .
$$

Applying the Cauchy product rule in the above equation and then equating the coefficients of like powers of $t$ in both sides of the resultant equation, assertion (2.11) follows.

Theorem 2.7. The following relation for the $q$-Hermite-based Frobenius-type Eulerian polynomials ${ }_{H} A_{n, q}^{(\alpha, s)}(x, y ; \lambda)$ of order $\alpha$ holds true:

$$
\begin{aligned}
H_{H} A_{n, q}^{(\alpha, s)}(x, y ; \lambda)= & \frac{1}{1-\lambda} \sum_{k=0}^{n}\left(\begin{array}{l}
n \\
k
\end{array}\right)_{q}\left[A_{n-k, q}(0, y ; \lambda)_{H} A_{k, q}^{(\alpha, s)}((\lambda-1) x, 0 ; \lambda)\right. \\
& \left.-\lambda A_{n-k, q}(0, y ; \lambda)_{H} A_{k, q}^{(\alpha, s)}(x, 0 ; \lambda)\right] .
\end{aligned}
$$

Proof. Consider generating function (2.1), we have

$$
\begin{gathered}
\sum_{n=0}^{\infty} H_{n, q}^{(\alpha, s)}(x, y ; \lambda) \frac{t^{n}}{[n]_{q} !} \\
=\left(\frac{1-\lambda}{e_{q}^{t(\lambda-1)}-\lambda}\right)\left(\frac{e_{q}^{t(\lambda-1)}-\lambda}{1-\lambda}\right)\left(\frac{1-\lambda}{e_{q}^{t(\lambda-1)}-\lambda}\right)^{\alpha} e_{q}\left(x t-\frac{s t^{2}}{1+q}\right) E_{q}(y t) \\
=\frac{1}{1-\lambda}\left(\frac{1-\lambda}{e_{q}^{t(\lambda-1)}-\lambda}\right) e_{q}^{(\lambda-1) t}\left(\frac{1-\lambda}{e_{q}^{t(\lambda-1)}-\lambda}\right)^{\alpha} e_{q}\left(x t-\frac{s t^{2}}{1+q}\right) E_{q}(y t)- \\
\frac{\lambda}{1-\lambda}\left(\frac{1-\lambda}{e_{q}^{t(\lambda-1)}-\lambda}\right)\left(\frac{1-\lambda}{e_{q}^{t(\lambda-1)}-\lambda}\right)^{\alpha} e_{q}\left(x t-\frac{s t^{2}}{1+q}\right) E_{q}(y t) \\
=\frac{1}{1-\lambda} \sum_{n=0}^{\infty} A_{n, q}(0, y ; \lambda) \frac{t^{n}}{[n]_{q} !} \sum_{k=0}^{\infty} A_{k, q}^{(\alpha, s)}((\lambda-1) x, 0 ; \lambda) \frac{t^{k}}{[k]_{q} !} \\
\quad-\frac{\lambda}{1-\lambda} \sum_{n=0}^{\infty} A_{n, q}(0, y ; \lambda) \frac{t^{n}}{[n]_{q} !} \sum_{k=0}^{\infty} H_{k, q}^{(\alpha, s)}(x, 0 ; \lambda) \frac{t^{k}}{[k]_{q} !} .
\end{gathered}
$$

Applying the Cauchy product rule in the above equation and then equating the coefficients of like powers of $t$ in both sides of the resultant equation, assertion (2.12) follows.

Theorem 2.8. The following recurrence relation for the $q$-Hermite-based Frobenius-type Eulerian polynomials ${ }_{H} A_{n, q}^{(\alpha, s)}(x, y ; \lambda)$ of order $\alpha$ holds true:

$$
\begin{gathered}
H A_{n+1, q}^{(s)}(x, y ; \lambda)=-\left(\frac{2 s}{1+q}\right)[n]_{q H} A_{n-1, q}^{(s)}(q x, q y ; \lambda)+x_{H} A_{n, q}^{(s)}(x, y ; \lambda) \\
+y_{H} A_{n, q}^{(s)}(q x, q y ; \lambda)+\frac{1}{(\lambda-1)^{2}} \sum_{k=0}^{n}\left(\begin{array}{l}
n \\
k
\end{array}\right)_{q} H_{n-k, q}^{(s)}(x, y ; \lambda) q^{n-k} A_{k, q}(1, \lambda-1 ; \lambda) .
\end{gathered}
$$

Proof. Taking $\alpha=1$ and then applying $q$-derivative on both sides of generating function (2.1), it follows that

$$
\sum_{n=0}^{\infty}{ }_{H} A_{n+1, q}^{(s)}(x, y ; \lambda) \frac{t^{n}}{[n]_{q} !}=(1-\lambda) D_{q, t}\left(\frac{e_{q}\left(x t-\frac{s t^{2}}{1+q}\right) E_{q}(y t)}{e_{q}^{t(\lambda-1)}-\lambda}\right)
$$


which on performing differentiation in left hand side, using formula (1.6), yields

$$
\begin{gathered}
\sum_{n=0}^{\infty} H_{n} A_{n+1, q}^{(s)}(x, y ; \lambda) \frac{t^{n}}{[n]_{q} !}=(1-\lambda)\left[\frac{\left(e_{q}^{q t(\lambda-1)}-\lambda\right) D_{q, t}\left(e_{q}(x t) e_{q}\left(-\frac{s t^{2}}{1+q}\right) E_{q}(y t)\right)}{\left(e_{q}^{t(\lambda-1)}-\lambda\right)\left(e_{q}^{q t(\lambda-1)}-\lambda\right)}\right] \\
-\frac{e_{q}(q x t) E_{q}(y q t) e_{q}\left(-\frac{s q^{2} t^{2}}{1+q}\right) D_{q, t}\left(e_{q}^{t(\lambda-1)}-\lambda\right)}{\left(e_{q}^{t(\lambda-1)}-\lambda\right)\left(e_{q}^{q t(\lambda-1)}-\lambda\right)} \\
=-\left(\frac{2 s}{1+q}\right)\left(\frac{1-\lambda}{e_{q}^{t(\lambda-1)}-\lambda}\right) e_{q}\left(q x t-\frac{s t^{2}}{1+q}\right) E_{q}(q y t) t \\
+x\left(\frac{1-\lambda}{e_{q}^{t(\lambda-1)}-\lambda}\right) e_{q}\left(x t-\frac{s t^{2}}{1+q}\right) E_{q}(y t)+y\left(\frac{1-\lambda}{e_{q}^{t(\lambda-1)}-\lambda}\right) e_{q}\left(q x t-\frac{s t^{2}}{1+q}\right) E_{q}(q y t) \\
+\frac{1}{(\lambda-1)^{2}}\left(\frac{1-\lambda}{e_{q}^{t(\lambda-1)}-\lambda}\right) e_{q}\left(q x t-\frac{s t^{2}}{1+q}\right) E_{q}(q y t)\left(\frac{1-\lambda}{e_{q}^{t(\lambda-1)}-\lambda}\right) e_{q}^{(\lambda-1) t},
\end{gathered}
$$

which on making use of the Cauchy product rule in the right hand side and comparing the coefficients of $\frac{t^{n}}{n !}$ on both sides of the resultant equation gives recurrence relation (2.13).

\section{Summation formulae for $q$-Hermite based Frobenius type Eulerian polynomials}

In this section, we provide implicit formulae, Stirling numbers of the second kind and some relationships for $q$-Hermite based Frobenius type Eulerian polynomials of order $\alpha$ related to Apostol type Bernoulli polynomials, Apostol type Euler polynomials and Apostol type Genocchi polynomials. We now begin with the following theorem.

Theorem 3.1. The following summation formulae for $q$-Hermite based Frobenius type Eulerian polynomials ${ }_{H} A_{n, q}^{(\alpha, s)}(x, y ; \lambda)$ of order $\alpha$ holds true:

$$
{ }_{H} A_{k+l, q}^{(\alpha, s)}(z, y ; \lambda)=\sum_{n, m=0}^{k, l}\left(\begin{array}{c}
l \\
m
\end{array}\right)_{q}\left(\begin{array}{l}
k \\
n
\end{array}\right)_{q}(z-x)^{n+m}{ }_{H} A_{k+l-n-m, q}^{(\alpha, s)}(x, y ; \lambda) .
$$

Proof. We replace $t$ by $t+w$ and rewrite the generating function (2.1) as

$$
\begin{aligned}
& \left(\frac{1-\lambda}{e_{q}((\lambda-1)(t+w)-\lambda)}\right)^{\alpha} E_{q}(y(t+w)) e_{q}\left(\frac{-s(t+u)^{2}}{1+q}\right) \\
= & \left.e_{q}(-x(t+w)) \sum_{k, l=0}^{\infty} H A_{k+l, q}^{(\alpha, s)}(x, y ; \lambda) \frac{t^{k}}{[k]_{q} !} \frac{w^{l}}{[l]_{q} !}, \quad \text { (see }[14,15]\right) .
\end{aligned}
$$

Replacing $x$ by $z$ in the above equation and equating the resulting equation to the above equation, we get

$$
\begin{gathered}
e_{q}((z-x)(t+w)) \sum_{k, l=0}^{\infty} H A_{k+l, q}^{(\alpha, s)}(x, y ; \lambda) \frac{t^{k}}{[k]_{q} !} \frac{w^{l}}{[l]_{q} !} \\
=\sum_{k, l=0}^{\infty} H A_{k+l, q}^{(\alpha, s)}(z, y ; \lambda) \frac{t^{k}}{[k]_{q} !} \frac{w^{l}}{[l]_{q} !} .
\end{gathered}
$$

On expanding exponential function (3.3) gives

$$
\sum_{N=0}^{\infty} \frac{[(z-x)(t+w)]^{N}}{[N]_{q} !} \sum_{k, l=0}^{\infty}{ }_{H} A_{k+l, q}^{(\alpha, s)}(x, y ; \lambda) \frac{t^{k}}{[k]_{q} !} \frac{w^{l}}{[l]_{q} !}
$$




$$
=\sum_{k, l=0}^{\infty} H_{H}^{(\alpha, s)}(z, y ; \lambda) \frac{t^{k}}{[k]_{q} !} \frac{w^{l}}{[l]_{q} !},
$$

which on using formula [18,p.52(2)]

$$
\sum_{N=0}^{\infty} f(N) \frac{(x+y)^{N}}{N !}=\sum_{n, m=0}^{\infty} f(n+m) \frac{x^{n}}{n !} \frac{y^{m}}{m !}
$$

in the left hand side becomes

$$
\begin{gathered}
\sum_{n, m=0}^{\infty} \frac{(z-x)^{n+m} t^{n} w^{m}}{[n]_{q} ![m]_{q} !} \sum_{k, l=0}^{\infty}{ }_{H} A_{k+l, q}^{(\alpha, s)}(x, y ; \lambda) \frac{t^{k}}{[k]_{q} !} \frac{w^{l}}{[l]_{q} !} \\
=\sum_{k, l=0}^{\infty} H A_{k+l, q}^{(\alpha, s)}(z, y ; \lambda) \frac{t^{k}}{[k]_{q} !} \frac{w^{l}}{[l]_{q} !}
\end{gathered}
$$

Now replacing $k$ by $k-n$, and $l$ by $l-m$ in the left hand side of (3.6), we get

$$
\begin{gathered}
\sum_{k, l=0}^{\infty} \sum_{n, m=0}^{k, l} \frac{(z-x)^{n+m}}{[n]_{q} ![m]_{q} !} A_{k+l-n-m, q}^{(\alpha, s)}(x, y ; \lambda) \frac{t^{k}}{(k-n)_{q} !} \frac{w^{l}}{(l-m)_{q} !} \\
=\sum_{k, l=0}^{\infty} H A_{k+l, q}^{(\alpha)}(z, y ; \lambda) \frac{t^{k}}{[k]_{q} !} \frac{w^{l}}{[l]_{q} !} .
\end{gathered}
$$

Finally on equating the coefficients of the like powers of $t$ and $w$ in the above equation, we get the required result.

Remark 3.1. By taking $l=0$ in Eq. (3.1), we immediately deduce the following result.

Corollary 3.1. The following summation formula for $q$-Hermite based Frobenius type Eulerian polynomials ${ }_{H} A_{n, q}^{(\alpha, s)}(x, y ; \lambda)$ of order $\alpha$ holds true:

$$
{ }_{H} A_{k+l, q}^{(\alpha, s)}(z, y ; \lambda)=\sum_{n=0}^{k}\left(\begin{array}{l}
k \\
n
\end{array}\right)_{q}(z-x)^{n}{ }_{H} A_{k-n, q}^{(\alpha, s)}(x, y ; \lambda) .
$$

Remark 3.2. On replacing $z$ by $z+x$ and setting $y=0$ in Theorem (3.1), we get the following result involving $q$-Hermite based Frobenius type Eulerian polynomials ${ }_{H} A_{n, q}^{(\alpha, s)}(x, y ; \lambda)$ of one variable

$$
{ }_{H} A_{k+l, q}^{(\alpha, s)}(z+x ; \lambda)=\sum_{n, m=0}^{k, l}\left(\begin{array}{c}
l \\
m
\end{array}\right)_{q}\left(\begin{array}{l}
k \\
n
\end{array}\right)_{q} z^{n+m}{ }_{H} A_{k+l-n-m, q}^{(\alpha, s)}(x ; \lambda),
$$

whereas by setting $z=0$ in Theorem 3.1, we get another result involving $q$-Hermite based Frobenius type Eulerian polynomials ${ }_{H} A_{n, q}^{(\alpha, s)}(x, y ; \lambda)$ of one and two variables

$$
{ }_{H} A_{k+l, q}^{(\alpha, s)}(y ; \lambda)=\sum_{n, m=0}^{k, l}\left(\begin{array}{c}
l \\
m
\end{array}\right)_{q}\left(\begin{array}{l}
k \\
n
\end{array}\right)_{q}(-x)^{n+m}{ }_{H} A_{k+l-n-m, q}^{(\alpha, s)}(x, y ; \lambda) .
$$

Theorem 3.2. The following summation formulae for $q$-Hermite based Frobenius type Eulerian polynomials ${ }_{H} A_{n, q}^{(\alpha, s)}(x, y ; \lambda)$ of order $\alpha$ holds true:

$$
{ }_{H} A_{n, q}^{(\alpha+1, s)}(x, y ; \lambda)=\sum_{m=0}^{n}\left(\begin{array}{c}
n \\
m
\end{array}\right){ }_{q} A_{n-m, q}(\lambda)_{H} A_{m, q}^{(\alpha, s)}(x, y ; \lambda) .
$$


Proof. From (2.1), we have

$$
\begin{aligned}
& \frac{1-\lambda}{e_{q}((\lambda-1) t)-\lambda}\left(\frac{1-\lambda}{e_{q}((\lambda-1) t)-\lambda}\right)^{\alpha} e_{q}\left(x t-\frac{s t^{2}}{1+q}\right) E_{q}(y t) \\
&= \frac{1-\lambda}{e_{q}((\lambda-1) t)-\lambda} \sum_{m=0}^{\infty} H_{m, q}^{(\alpha, s)}(x, y ; \lambda) \frac{t^{m}}{[m]_{q} !} \\
& \sum_{n=0}^{\infty}{ }_{H} A_{n, q}^{(\alpha+1, s)}(x, y ; \lambda) \frac{t^{n}}{[n]_{q} !}=\sum_{n=0}^{\infty} A_{n, q}(\lambda) \frac{t^{n}}{[n]_{q} !} \sum_{m=0}^{\infty} H_{m, q}^{(\alpha, s)}(x, y ; \lambda) \frac{t^{m}}{[m]_{q} !} .
\end{aligned}
$$

Now replacing $n$ by $n-m$ and equating the coefficients of $t^{n}$ leads to formula (3.11).

Theorem 3.3. The following summation formulae for $q$-Hermite based Frobenius type Eulerian polynomials ${ }_{H} A_{n, q}^{(\alpha, s)}(x, y ; \lambda)$ of order $\alpha$ holds true:

$$
{ }_{H} A_{n, q}^{(\alpha, s)}(x+1, y ; \lambda)=\sum_{k=0}^{n}\left(\begin{array}{l}
n \\
k
\end{array}\right)_{q} A_{k, q}^{(\alpha, s)}(x, y ; \lambda)
$$

Proof. Using definition (2.1), we have

$$
\begin{gathered}
\sum_{n=0}^{\infty}{ }_{H} A_{n}^{(\alpha, s)}(x+1, y ; \lambda) \frac{t^{n}}{n !}=\left(\frac{1-\lambda}{e_{q}((\lambda-1) t)-\lambda}\right)^{\alpha} e_{q}\left((x+1) t-\frac{s t^{2}}{1+q}\right) E_{q}(y t) \\
=\left(\frac{1-\lambda}{e_{q}((\lambda-1) t)-\lambda}\right)^{\alpha} e_{q}\left(x t-\frac{s t^{2}}{1+q}\right) E_{q}(y t) e_{q}(t) \\
=\left(\sum_{k=0}^{\infty} H_{k, q}^{(\alpha, s)}(x, y ; \lambda) \frac{t^{k}}{[k]_{q} !}\right)\left(\sum_{n=0}^{\infty} \frac{t^{n}}{[n]_{q} !}\right) \\
=\sum_{n=0}^{\infty}\left(\sum_{k=0}^{n}\left(\begin{array}{l}
n \\
k
\end{array}\right)_{q} A_{k, q}^{(\alpha, s)}(x, y ; \lambda)\right) \frac{t^{n}}{[n]_{q} !}
\end{gathered}
$$

Finally, equating the coefficients of the like powers of $t^{n}$, we get (3.12).

The $q$-Stirling numbers of the second kind is defined by

$$
\sum_{n=0}^{\infty} S_{2, q}(k, n) \frac{t^{n}}{[n]_{q} !}=\frac{\left(e_{q}(t)-1\right)^{k}}{[k]_{q} !}, \text { see }[10-13,16]
$$

Theorem 3.4. The following relationship

$$
{ }_{H} A_{n, q}^{(\alpha, s)}(x, y ; \lambda)=\sum_{u=0}^{n} \sum_{k=0}^{n}\left(\begin{array}{l}
n \\
u
\end{array}\right)_{q}[k]_{q} !\left(\begin{array}{c}
-\alpha \\
k
\end{array}\right)_{q}(1-\lambda)^{k} S_{2, q}(k, u)(\lambda-1)^{u} H_{n-u, q}^{(s)}(x, y)
$$

holds true. 
10

Proof. From (2.1), we have

$$
\begin{gathered}
\sum_{n=0}^{\infty}{ }_{H} A_{n, q}^{(\alpha, s)}(x, y ; \lambda) \frac{t^{n}}{[n]_{q} !}=\left(\frac{1-\lambda}{e_{q}((\lambda-1) t)-\lambda}\right)^{\alpha} e_{q}\left(x t-\frac{s t^{2}}{1+q}\right) E_{q}(y t) \\
=e_{q}\left(x t-\frac{s t^{2}}{1+q}\right) E_{q}(y t)\left(1+\frac{e_{q}((\lambda-1) t)-1}{1-\lambda}\right)^{-\alpha} \\
=\sum_{k=0}^{\infty}\left(\begin{array}{c}
-\alpha \\
k
\end{array}\right)\left(\frac{e_{q}((\lambda-1) t)-1}{1-\lambda}\right)^{k} \sum_{n=0}^{\infty} H_{n, q}^{(s)}(x, y) \frac{t^{n}}{[n]_{q} !} \\
=\sum_{n=0}^{\infty}\left(\sum_{u=0}^{n} \sum_{k=0}^{n}\left(\begin{array}{l}
n \\
u
\end{array}\right)_{q}[k]_{q} !\left(\begin{array}{c}
-\alpha \\
k
\end{array}\right)(1-\lambda)^{k} S_{2, q}(k, u)(\lambda-1)^{u} H_{n-u, q}^{(s)}(x, y)\right) \frac{t^{n}}{[n]_{q} !}
\end{gathered}
$$

On comparing the coefficients of $t^{n}$ in both sides, we get (3.13).

Theorem 3.5. The following relationship

$$
{ }_{H} A_{n, q}^{(\alpha, s)}(x, y ; \lambda)=\sum_{u=0}^{n} \sum_{k=0}^{n}\left(\begin{array}{l}
n \\
u
\end{array}\right)_{q}[k]_{q} !\left(\begin{array}{c}
\alpha+k-1 \\
k
\end{array}\right) S_{2, q}(k, u)(\lambda-1)^{u+k} H_{n-u, q}^{(s)}(x, y)
$$

holds true.

Proof. By using generating function (2.1), we have

$$
\begin{gathered}
\sum_{n=0}^{\infty}{ }_{H} A_{n, q}^{(\alpha, s)}(x, y ; \lambda) \frac{t^{n}}{[n]_{q} !}=\left(\frac{1-\lambda}{e_{q}((\lambda-1) t)-\lambda}\right)^{\alpha} e_{q}\left(x t-\frac{s t^{2}}{1+q}\right) E_{q}(y t) \\
=e_{q}\left(x t-\frac{s t^{2}}{1+q}\right) E_{q}(y t)\left(1+\frac{e_{q}((\lambda-1) t)-1}{1-\lambda}\right)^{-\alpha} \\
=\sum_{k=0}^{\infty}(-1)^{k}\left(\begin{array}{c}
\alpha+k-1 \\
k
\end{array}\right)\left(\frac{e_{q}((\lambda-1) t)-1}{1-\lambda}\right)^{k} \sum_{n=0}^{\infty} H_{n, q}^{(s)}(x, y) \frac{t^{n}}{[n]_{q} !} \\
=\sum_{n=0}^{\infty}\left(\sum_{u=0}^{n} \sum_{k=0}^{n}\left(\begin{array}{l}
n \\
u
\end{array}\right)_{q}[k]_{q} !\left(\begin{array}{c}
\alpha+k-1 \\
k
\end{array}\right) S_{2, q}(k, u)(\lambda-1)^{u+k} H_{n-u, q}^{(s)}(x, y)\right) \frac{t^{n}}{[n]_{q} !}
\end{gathered}
$$

Comparing the coefficients of $t^{n}$ in both sides, we arrive at the required result (3.14).

Theorem 3.6. The following relation between the $q$-Hermite based Frobenius type Eulerian polynomials ${ }_{H} A_{n, q}^{(\alpha, s)}(x, y ; \lambda)$ and Apostol type $q$-Bernoulli polynomials $B_{n, q}(x ; \lambda)$ holds true:

$$
\begin{aligned}
{ }_{H} A_{n, q}^{(\alpha, s)}(x, y ; \lambda)=\frac{1}{[n+1]_{q}} \sum_{k=0}^{n+1}\left(\begin{array}{c}
n+1 \\
k
\end{array}\right)_{q}\left(\lambda \sum_{r=0}^{k}\left(\begin{array}{l}
k \\
r
\end{array}\right)_{q} B_{k-r, q}(x ; \lambda)-B_{k, q}(x ; \lambda)\right) \\
\times{ }_{H} A_{n-k+1, q}^{(\alpha, s)}(0, y ; \lambda) .
\end{aligned}
$$

Proof. Consider generating function (2.1), we have

$$
\begin{gathered}
\sum_{n=0}^{\infty} H_{H} A_{n, q}^{(\alpha, s)}(x, y ; \lambda) \frac{t^{n}}{[n]_{q} !} \\
=\left(\frac{1-\lambda}{e_{q}(t(\lambda-1))-\lambda}\right)^{\alpha} e_{q}\left(x t-\frac{s t^{2}}{1+q}\right) E_{q}(y t)\left(\frac{t}{\lambda e_{q}(t)-1}\right)\left(\frac{\lambda e_{q}(t)-1}{t}\right) \\
=\frac{1}{t}\left(\lambda \sum_{n=0}^{\infty} H A_{n, q}^{(\alpha, s)}(0, y ; \lambda) \frac{t^{n}}{[n]_{q} !} \sum_{k=0}^{\infty} B_{k, q}(x ; \lambda) \frac{t^{k}}{[k]_{q} !} \sum_{r=0}^{\infty} \frac{t^{r}}{[r]_{q} !}\right.
\end{gathered}
$$




$$
\left.-\sum_{n=0}^{\infty}{ }_{H} A_{n, q}^{(\alpha, s)}(0, y ; \lambda) \frac{t^{n}}{[n]_{q} !} \sum_{k=0}^{\infty} B_{k, q}(x ; \lambda) \frac{t^{k}}{[k]_{q} !}\right) .
$$

On equating the coefficients of same powers of $t$ after using Cauchy product rule in (3.16), assertion (3.15) follows.

Theorem 3.7. The following relation between the $q$-Hermite based Frobenius type Eulerian polynomials ${ }_{H} A_{n, q}^{(\alpha, s)}(x, y ; \lambda)$ and Apostol type Euler polynomials $E_{n, q}(x ; \lambda)$ holds true:

$$
\begin{aligned}
{ }_{H} A_{n, q}^{(\alpha, s)}(x, y ; \lambda)=\frac{1}{2} \sum_{k=0}^{n} & \left(\begin{array}{l}
n \\
k
\end{array}\right)_{q}\left(\lambda \sum_{r=0}^{k}\left(\begin{array}{l}
k \\
r
\end{array}\right)_{q} E_{k-r, q}(x ; \lambda)+E_{k, q}(x ; \lambda)\right) \\
& \times{ }_{H} A_{n-k, q}^{(\alpha, s)}(0, y ; \lambda) .
\end{aligned}
$$

Proof. Consider generating function (2.1), we have

$$
\begin{gathered}
\sum_{n=0}^{\infty}{ }_{H} A_{n, q}^{(\alpha)}(x, y ; \lambda) \frac{t^{n}}{[n]_{q} !} \\
=\left(\frac{1-\lambda}{e_{q}(t(\lambda-1))-\lambda}\right)^{\alpha} e_{q}\left(x t-\frac{s t^{2}}{1+q}\right) E_{q}(y t)\left(\frac{2}{\lambda e_{q}(t)+1}\right)\left(\frac{\lambda e_{q}(t)+1}{2}\right) \\
=\frac{1}{2}\left(\lambda \sum_{n=0}^{\infty} H A_{n, q}^{(\alpha, s)}(0, y ; \lambda) \frac{t^{n}}{[n]_{q} !} \sum_{k=0}^{\infty} E_{k, q}(x ; \lambda) \frac{t^{k}}{[k]_{q} !} \sum_{r=0}^{\infty} \frac{t^{r}}{[r]_{q} !}\right. \\
\left.+\sum_{n=0}^{\infty} H_{H} A_{n, q}^{(\alpha, s)}(0, y ; \lambda) \frac{t^{n}}{[n]_{q} !} \sum_{k=0}^{\infty} E_{k, q}(x ; \lambda) \frac{t^{k}}{[k]_{q} !}\right) .
\end{gathered}
$$

On equating the coefficients of same powers of $t$ after using Cauchy product rule in (3.18), assertion (3.17) follows.

Theorem 3.8. The following relation between the $q$-Hermite based Frobenius type Eulerian polynomials ${ }_{H} A_{n, q}^{(\alpha, s)}(x, y ; \lambda)$ and Apostol type Genocchi polynomials $G_{n, q}(x ; \lambda)$ holds true:

$$
\begin{gathered}
{ }_{H} A_{n, q}^{(\alpha, s)}(x, y ; \lambda)=\frac{1}{2[n+1]_{q}} \sum_{k=0}^{n+1}\left(\begin{array}{c}
n+1 \\
k
\end{array}\right)_{q}\left(\lambda \sum_{r=0}^{k}\left(\begin{array}{c}
k \\
r
\end{array}\right)_{q} G_{k-r, q}(x ; \lambda)+G_{k, q}(x ; \lambda)\right) \\
\times_{H} A_{n-k+1, q}^{(\alpha, s)}(0, y ; \lambda) .
\end{gathered}
$$

Proof. Consider generating function (2.1), we have

$$
\begin{gathered}
\sum_{n=0}^{\infty} H_{H, q} A_{n, q}^{(\alpha)}(x, y ; \lambda) \frac{t^{n}}{[n]_{q} !} \\
=\left(\frac{1-\lambda}{e_{q}(t(\lambda-1))-\lambda}\right)^{\alpha} e_{q}\left(x t-\frac{s t^{2}}{1+q}\right) E_{q}(y t)\left(\frac{2 t}{\lambda e_{q}(t)+1}\right)\left(\frac{\lambda e_{q}(t)+1}{2 t}\right) \\
=\frac{1}{2 t}\left(\lambda \sum_{n=0}^{\infty} H A_{n, q}^{(\alpha, s)}(0, y ; \lambda) \frac{t^{n}}{[n]_{q} !} \sum_{k=0}^{\infty} G_{k, q}(x ; \lambda) \frac{t^{k}}{[k]_{q} !} \sum_{r=0}^{\infty} \frac{t^{r}}{[r]_{q} !}\right. \\
\left.+\sum_{n=0}^{\infty} H A_{n, q}^{(\alpha, s)}(0, y ; \lambda) \frac{t^{n}}{[n]_{q} !} \sum_{k=0}^{\infty} G_{k, q}(x ; \lambda) \frac{t^{k}}{[k]_{q} !}\right) .
\end{gathered}
$$

On equating the coefficients of same powers of $t$ after using Cauchy product rule in (3.20), assertion (3.19) follows. 


\section{Conclusion}

In the present paper, we have initially considered a novel kind of the Hermite based Frobenius type Eulerian polynomials based on $q$-numbers and then have previded multifarious correlation, implicit summation formula, identities, explicit formulas and recurrence relations for the aforementioned polynomials by means of the series manipulation methods and some special techniques. The aforegoing polynomials involves a lot of well known special polynomials in literature. Furthermore, we have given several relationships covering the $q$ Apostol-Bernoulli polynomials, $q$-Apostol-Euler polynomials, $q$-Apostol-Genocchi polynomials and $q$-Stirling numbers of the second kind associated with the $q$-Hermite based Frobenius type Eulerian polynomials.

\section{References}

[1] Al-Salam, W. A, q-Appell polynomials, Ann. Mat. Pura Appl., (4)17(1967), 31-45.

[2] Andrews, G. E, Akey, R, Roy, R, Special functions, Cambridge University Press, Cambridge, 1999.

[3] Carlitz, L, Eulerian numbers and polynomials, Math. Mag., 32(1959), 247-260.

[4] Carlitz, L, Eulerian numbers and polynomials of higher order, Duke Math. J., 27(1960), $401-423$.

[5] Cheon, G. S, Jung, J. H, The $q$-Sheffer sequence of a new type and associated orthogonal polynomials, Linear Algebra Appl., 491(2016), 247-260.

[6] Kim, D. S, Kim, T, Kim, Y. H, Dolgy, D. V, A note on Eulerian polynomials associated with Bernoulli and Euler numbers and polynomials, Adv. Stud. Contemp. Math., 22(2012), 379-389.

[7] Kim, D. S, Kim, T, Kim, W. J, Dolgy, D. V, A note on Eulerian polynomials, Abtr. Appl. Anal. (2012) Art. ID 269640, 10pp.

[8] Keleshteri, M. E, Mahmudov, N. I, A study on $q$-Appell polynomials from determinantal point of view, Appl. Math. Comput., 260(2015), 351-369.

[9] Keleshteri, M. E, Mahmudov, N. I, On the class of 2D q-Appell polynomials, arXiv:1512.03255v1.

[10] Mahmudov, N. I, On a class of $q$-Bernoulli and $q$-Euler polynomials, Adv. Differ. Equ., 108(2013), $1-11$.

[11] Mahmudov, N. I, Difference equations of $q$-Appell polynomials, Appl. Math. Comput., 245(2014), 539-543.

[12] Mahmudov, N. I, Keleshteri, M. E, q-extensions for the Apostol type polynomials, J. Appl. Math., (2014) Art. ID 868167, 1-8.

[13] Mahmudov, N. I, Momenzadeh, M, On a class of $q$-Bernoulli, $q$-Euler and $q$-Genocchi polynomials, Abstr. Appl. Anal., (2014), Art. ID 696454, 1-10.

[14] Pathan, M. A and Khan, W. A, Some implicit summation formulas and symmetric identities for the generalized Hermite-Bernoulli polynomials, Mediterr. J. Math., 12(2015), 679-695.

[15] Pathan, M. A and Khan, W. A, A new class of generalized polynomials associated with Hermite and Euler polynomials, Mediterr. J. Math., 13(2016), 913-928.

[16] Riyasat, M, Khan, S, Some results on $q$-Hermite based hybrid polynomials, Glasnik Matematicki, $53(73)(2018), 9-31$.

[17] Srivastava, H. M, Eulerian and other integral representations for some families of hypergeometric polynomials, Inter. J. Appl. Math. Stat., 11(2007), 149-171.

[18] Srivastava, H. M and Manocha, H. L, A treatise on generating functions, Ellis Horwood Limited, New York, 1984.

[19] Srivastva, H. M, Boutiche, M. A, Rahmani, M, A class of Frobenius-type Eulerian polynomials, Rocky Mountain J. Math., 48(2018), 1003-1013. 